\title{
GROMOV-FLOER THEORY AND DISJUNCTION ENERGY OF COMPACT LAGRANGIAN EMBEDDINGS
}

\author{
YONG-GEUN OH
}

\begin{abstract}
In this paper, we give a new simple proof of Chekanov's positivity theorem of the disjunction energy of compact Lagrangian submanifolds in tame symplectic manifolds. As a consequence, it also gives rise to a simple proof of nondegeneracy of Hofer's norm on the group of Hamiltonian diffeomorphisms on any tame symplectic manifolds.
\end{abstract}

\section{Introduction}

To put the method we use in the present paper in perspective, we first summarize the history of previous works related to this paper.

In [H2], Hofer introduced a bi-invariant norm on the group of Hamiltonian diffeomorphisms on symplectic manifold $(P, \omega)$ : For compactly supported Hamiltonian diffeomorphism $H: P \times[0,1] \rightarrow \mathbb{R}$, Hofer's norm of $\|H\|$ is defined to be

$$
\|H\|=\int_{0}^{1}\left(\max H_{t}-\min H_{t}\right) d t
$$

and for a Hamiltonian diffeomorphism $\phi$, its norm is defined to be

$$
\|\phi\|=\inf _{H \mapsto \phi}\|H\|
$$

where $H \mapsto \phi$ means that $\phi=\phi_{H}^{1}$. Here $\phi_{H}^{1}$ denotes the time-one map of the flow of the Hamilton's equation

$$
\dot{z}=X_{H}(z)
$$

While invariance and triangle inequality are immediate consequences from its definition and from some simple calculations in Hamiltonian dynamics (see [H2]), nondegeneracy of the norm is a highly nontrivial fact which encodes the $C^{0}$ rigidity in symplectic geometry in a remarkable way. In the same paper, Hofer

Received February 28, 1997.

This research is partially supported by NSF grant \# DMS-9504455. 
proved the nondegeneracy on $\mathbb{C}^{n}$, with a delicate variational theory of Hamiltonian systems, by comparing the disjunction energy with a symplectic capacity of the Euclidean ball in $\mathbb{C}^{n}$.

A symplectic manifold $(P, \omega)$ is called tame if it allows an almost complex structure $J_{0}$ such that the bilinear form $\omega\left(\cdot, J_{0} \cdot\right)$ defines a complete Riemannian metric on $P$ with bounded curvature and with injectivity radius bounded away from zero. In this case, we also call tame the triple $\left(P, \omega, J_{0}\right)$ or the almost complex structure $J_{0}$. As usual, when we do our estimates which are implicit mostly in this paper, we will use various norms always in terms of a fixed such metric.

In $[\mathrm{P}]$, Polterovich proved the nondegeneracy on any tame rational symplectic manifolds by studying disjunction energy of Lagrangian submanifolds via Gromov's theory of pseudo-holomorphic curves : For a given Lagrangian submanifold $L \subset(P, \omega)$, its disjunction energy is defined by

$$
E(L: P, \omega)=\inf _{H}\left\{\|H\| \mid L \cap \phi_{H}^{1}(L)=\emptyset\right\}
$$

In the same paper $[\mathrm{P}]$, Polterovich proved that

$$
E(L: P, \omega) \geq \frac{1}{2} \Gamma_{(L: P, \omega)}>0
$$

where $\Gamma_{(L: P, \omega)}$ is the (positive) generator of the subgroup

$$
\left\{\omega(B) \mid B \in \pi_{2}(P, L)\right\} \subset \mathbb{R}
$$

for any rational Lagrangian submanifold: $L \subset(P, \omega)$ is called rational if this subgroup of $\mathbb{R}$ is discrete. It is an easy consequence from this to obtain nondegeneracy of the norm in (1.2) for tame rational symplectic manifolds (see $[\mathrm{P}]$ for details). Via a version of the Floer homology theory, Chekanov [C1] improved this result by removing the factor " $\frac{1}{2}$ " in (1.4) and also by giving an estimate of the number of intersections $L \cap \phi_{H}^{1}(L)$ in relation to Arnold's conjecture.

In [LM], Lalonde-McDuff studied again the disjunction energy of symplectic balls as in [H2] and proved the non-degeneracy on arbitrary symplectic manifolds. In this paper, they used elaborate techniques of pseudo-holomorphic curves together with an ingenious "wrapping" construction of symplectic embedding of balls and proved that non-degeneracy of the norm is equivalent to non-squeezing theorem in arbitrary symplectic manifolds which they also proved in [LM].

Generalizing the results from $[\mathrm{P}]$ and $[\mathrm{C} 1]$, Chekanov $[\mathrm{C} 2]$ proved a positivity theorem of disjunction energy of any compact Lagrangian embeddings in tame symplectic manifolds by an even more delicate version of Floer homology theory than the one in [C1].

The main purpose of this paper is to give a new simple proof of this last theorem using a natural set-up of Gromov-Floer theory of perturbed CauchyRiemann equations. We first need some preliminary definitions to state 
Chekanov's theorem. As in [O4] or [C2], we define for each tame $J_{0}$,

$$
\begin{aligned}
A_{\left(J_{0}: P, \omega\right)} & =\inf \left\{\omega(v) \mid v: S^{2} \rightarrow P, \text { non-constant and } \bar{\partial}_{J_{0}} v=0\right\} \\
A_{\left(J_{0}, L: P, \omega\right)} & =\inf \left\{\omega(w) \mid w:\left(D^{2}, \partial D^{2}\right) \rightarrow(P, L), \text { non-constant and } \bar{\partial}_{J_{0}} w=0\right\} .
\end{aligned}
$$

It is not difficult to show (see [Corollary 3.5, O1] for its proof) that tameness of $\left(P, \omega, J_{0}\right)$ implies

$$
A_{\left(J_{0}: P, \omega\right)}, A_{\left(J_{0}, L: P, \omega\right)}>0
$$

We then define

$$
A_{(L: P, \omega)}=\sup _{J_{0}} \min \left\{A_{\left(J_{0}: P, \omega\right)}, A_{\left(J_{0}, L: P, \omega\right)}\right\} .
$$

Using this quantity, Chekanov [C2] proved the following theorem

Theorem [Chekanov, C2]. Let $(P, \omega)$ be a tame symplectic manifold and $L \subset(P, \omega)$ be a compact Lagrangian embedding. Then we have

$$
E(L: P, \omega) \geq A_{(L: P, \omega)} .
$$

The method we use in this paper is an outgrowth of many people's works, most notably Gromov's [G], Floer-Hofer-Viterbo's [FHV], Polterovich's [P] and the author's [O4-6]. We use the cut-off version (2.4) of the standard perturbed Cauchy-Riemann equation used in the dynamical version of the Floer theory in the following way: We first identify emptiness of intersections of two Lagrangian submanifolds $L$ and $\phi_{H}^{1}(L)$ as the obstruction to compactness of certain parametrized moduli space of perturbed Cauchy-Riemann equations. We then combine some simple calculation to relate the disjunction energy and the quantity $A_{(L: P, \omega)}$. Starting from [H1], several crude forms of this calculation have been implicitly used in many literature (see e.g., p. 979 of [O2]) on the Floer homology in symplectic geometry. In this paper, we carry out the optimal form of this calculation, which will quite easily give rise to the above theorem in our set-up. This kind of optimal form of calculations used in this paper first appeared in [C1] in a rather ambiguous way, and has been systematically used in our previous papers $[\mathrm{O} 5,6]$.

As in $[\mathrm{P}]$ and $[\mathrm{C} 1,2]$, we would like to mention that Chekanov's theorem immediately implies that Hofer's norm on the group of Hamiltonian diffeomorphisms of tame symplectic manifolds is nondegenerate. We suspect that a more careful analysis of our arguments will give rise to the proof of this nondegeneracy in any (tame or not) symplectic manifold as proven in [LM]. We will refine the analysis of bubblings more carefully and study the Maslov class obstruction to compact Lagrangian embeddings elsewhere. 


\section{Cut-off perturbed Cauchy-Riemann equations}

The materials we use in this section is partially influenced by Section 5 in Fukaya-Ono's paper [FO] in the context of fixed points of Hamiltonian diffeomorphisms, which in turn is similar to the arguments by Floer-Hofer-Viterbo in $[\mathrm{FHV}]$ in their proof of Weinstein's conjecture.

For each $K \in \mathbb{R}_{+}=[0, \infty)$, we define a function $\rho_{K}: \mathbb{R} \rightarrow[0,1]$ as follows: for $K \geq 1$, we define

$$
\rho_{K}(\tau)= \begin{cases}0 & \text { for }|\tau| \geq K+1 \\ 1 & \text { for }|\tau| \leq K\end{cases}
$$

with

$$
\begin{aligned}
& \rho_{K}^{\prime}<0 \text { for } K<\tau<K+1 \\
& >0 \text { for }-K-1<\tau<-K
\end{aligned}
$$

and for $0 \leq K \leq 1$,

$$
\rho_{K}=K \cdot \rho_{1}
$$

In particular, $\rho_{0} \equiv 0$.

Let $H: P \times[0,1] \rightarrow \mathbb{R}$ be a Hamiltonian such that

$$
\phi_{H}^{1}(L) \cap L=\emptyset,
$$

i.e., such that the equation

$$
\left\{\begin{array}{l}
\dot{z}=X_{H}(z) \\
z(0), z(1) \in L
\end{array}\right.
$$

has no solutions, and $J=\left\{J_{(\tau, t)}\right\}_{(\tau, t) \in \mathbb{R} \times[0,1]}$ be a two parameter family of tamed almost complex structure such that

$$
J_{(\tau, t)} \equiv J_{0} \quad \text { for }|\tau| \text { sufficiently large or for } t=0,1
$$

where $J_{0}$ is a fixed (genuine) almost complex structure on $P$ that is tamed to $\omega$.

We would like to remark that it is necessary to vary almost complex structures in terms of $t$ to get appropriate transversality result for the Floer complex (see [FHS], [O3] for detailed account of the transversality proof).

Throughout this paper, we will exclusively denote by $J_{0}$ any (genuine) almost complex structure and by $J$ a two-parameter version of them. We denote a one-parameter family of them by

$$
\bar{J}=\left\{J_{K}\right\}_{K \in[0,+\infty)}
$$

such that $J_{0}(\tau, t, m) \equiv J_{0}(m)$ is a time-independent almost complex structure and $J_{K}=J_{\infty}$ for sufficiently large $K$. 
For each such pair $(\bar{J}, H)$ and for $K \in \mathbb{R}_{+}$, we consider one parameter family of perturbed Cauchy-Riemann equations

$$
\left\{\begin{array}{l}
\frac{\partial u}{\partial \tau}+J_{K}(\tau, t, u)\left(\frac{\partial u}{\partial t}-\rho_{K}(\tau) X_{H}(u)\right)=0 \\
u(\tau, 0), u(\tau, 1) \in L
\end{array}\right.
$$

where $u: \mathbb{R} \times[0,1] \rightarrow P$. This equation should be regarded as the one used for the chain isotopy in the Floer homology theory connecting the Hamiltonian 0 to $H$ and then to 0 back. We will be interested in the solutions of (2.4) with finite energy

$$
E_{J_{K}}(u):=\int_{-\infty}^{\infty} \int_{0}^{1}\left|\frac{\partial u}{\partial \tau}\right|_{J_{K}(\tau, t, u)}^{2} d t d \tau<\infty .
$$

Noting that $\mathbb{R} \times[0,1]$ is conformally isomorphic to $D^{2} \backslash\{-1,1\}$, it follows that (2.4) and (2.5) imply that the map

$$
u \circ \phi:\left(D^{2} \backslash\{-1,1\}, \partial D^{2} \backslash\{-1,1\}\right) \rightarrow(P, L),
$$

where $\phi$ is a conformal map, has finite (harmonic) energy and $J_{0}$-holomorphic near $\{-1,1\}$. Then the removable singularity theorem [O1] enables us to extend this to the whole disc, which we denote by

$$
\widetilde{u}:\left(D^{2}, \partial D^{2}\right) \rightarrow(P, L)
$$

and which becomes a perturbed $J_{0}$-holomorphic disc. We denote by $[u] \in$ $\pi_{2}(P, L)$ the homotopy class defined by $\widetilde{u}$.

Now for each $K \in \mathbb{R}_{+}$and for $A \in \pi_{2}(P, L)$, we study the following moduli space

$$
\begin{gathered}
\mathcal{M}_{K}^{A}(\bar{J}, H)=\left\{u: \mathbb{R} \times[0,1] \rightarrow P \mid u \text { satisfies }(2.4), E_{J_{K}}(u)<\infty\right. \\
\text { and } \left.[u]=A \text { in } \pi_{2}(P, L)\right\} .
\end{gathered}
$$

Since (2.4) is a compact perturbation of the standard pseudo-holomorphic equation of discs with Lagrangian boundary condition, the standard index formula from $[\mathrm{G}]$ implies

$$
\operatorname{dim} \mathcal{M}_{K}^{A}(\bar{J}, H)=\mu_{L}(A)+n
$$

for generic $J, H$, provided it is non-empty. Here $n$ denotes the dimension of the Lagrangian submanifold $L$.

Lemma 2.1. $\mathcal{M}_{0}^{A}(\bar{J}, H)$ for $A=0$ in $\pi_{2}(P, L)$ consists of constant solutions and so $\mathcal{M}_{0}^{0}(\bar{J}, H)$ is diffeomorphic to L. Furthermore $\mathcal{M}_{0}^{0}(\bar{J}, H)$ is Fredholm regular for any almost complex structure $J_{0}$. 
Proof. For $K=0,(2.3)$ becomes

$$
\frac{\partial u}{\partial \tau}+J_{0} \frac{\partial u}{\partial t}=0
$$

Since $[u]=0, u$ must be constant. The Fredholm regularity of constant solutions is not difficult to check and is well-known to experts. We omit its proof.

Lemma 2.2. For any given $(\bar{J}, H)$ satisfying (2.1) and (2.2), there exists a constant $K_{0}>0$ such that $\mathcal{M}_{K}^{0}(\bar{J}, H)=\emptyset$ for all $K \in \mathbb{R}_{+}$with $K \geq K_{0}$.

Proof. First we prove a simple a priori energy bound for any element $u: \mathbb{R} \times$ $[0,1] \rightarrow P$ in (2.6). A straightforward calculation shows

$$
\begin{aligned}
E_{J_{K}}(u) & =\int_{0}^{1} \int_{-\infty}^{\infty}\left|\frac{\partial u}{\partial \tau}\right|_{J_{K}}^{2} d \tau d t=\iint \omega\left(\frac{\partial u}{\partial \tau}, J_{K} \frac{\partial u}{\partial \tau}\right) d \tau d t \\
& =\iint \omega\left(\frac{\partial u}{\partial \tau}, \frac{\partial u}{\partial t}-\rho_{K}(\tau) X_{H}(u)\right) d \tau d t \\
& =\iint \omega\left(\frac{\partial u}{\partial \tau}, \frac{\partial u}{\partial t}\right) d \tau d t-\iint \rho_{K}(\tau) \omega\left(\frac{\partial u}{\partial \tau}, X_{H}(u)\right) d \tau d t
\end{aligned}
$$

For the first term, we have

$$
\iint \omega\left(\frac{\partial u}{\partial \tau}, \frac{\partial u}{\partial t}\right) d \tau d t=\int u^{*} \omega=0
$$

because of the assumptions that $E_{J}(u)<\infty$ so that $u$ can be compactified as above and that $[u]=0$ in $\pi_{2}(P, L)$. For the second term, we have

$$
\begin{aligned}
-\int & \int \rho_{K}(\tau) \omega\left(\frac{\partial u}{\partial \tau}, X_{H}(u)\right) d \tau d t=\int_{-\infty}^{\infty} \rho_{K}(\tau) \int_{0}^{1} d H\left(\frac{\partial u}{\partial \tau}\right) d \tau d t \\
& =\int_{-\infty}^{\infty} \rho_{K}(\tau) \int_{0}^{1} \frac{\partial}{\partial \tau}(H \circ u) d t d \tau \\
& =-\int_{-\infty}^{\infty} \rho_{K}^{\prime}(\tau) \int_{0}^{1} H(u) d t d \tau \\
& \leq-\int_{-K-1}^{-K} \rho_{K}^{\prime}(\tau)\left(\int_{0}^{1} \max H_{t} d t\right) d \tau-\int_{K}^{K+1} \rho_{K}^{\prime}(\tau)\left(\int_{0}^{1} \max H_{t} d t\right) d \tau \\
& \leq \int_{0}^{1}\left(\max H_{t}-\min H_{t}\right) d t=:\|H\|
\end{aligned}
$$

where $\|H\|$ is Hofer's norm of $H$. The first inequality above holds because we have $\rho_{K}^{\prime} \geq 0$ on $[-K,-K+1]$ (resp. $\rho_{K}^{\prime} \leq 0$ on $\left.[K, K+1]\right)$, and $\rho_{K}^{\prime} \equiv 0$ otherwise. Hence we have proven

$$
E_{J_{K}}(u) \leq\|H\|
$$


for any $J, u \in \mathcal{M}_{K}^{0}(\bar{J}, H)$ and for all $K \in \mathbb{R}_{+}$.

Remark 2.3. If $[u]=A \in \pi_{2}(P, L)$, then (2.8) will be replaced by

$$
E_{J_{K}}(u) \leq \omega(A)+\|H\| .
$$

The inequality (2.8) will be crucial in our proof of positivity theorem of the disjunction energy of Lagrangian embeddings.

Now we go back to the proof of Lemma 2.2. Suppose the contrary that there exists $K_{j} \rightarrow \infty$ such that $\mathcal{M}_{K_{j}}^{0}(\bar{J}, H) \neq \emptyset$. Let $u_{j} \in \mathcal{M}_{K_{j}}^{0}(\bar{J}, H)$. Using the a priori bound (2.8), by taking a subsequence and taking away bubblings if necessary, we find a local limit

$$
u_{0}: \mathbb{R} \times[0,1] \rightarrow P
$$

that satisfies the energy bound $E_{J}\left(u_{0}\right) \leq\|H\|$, in particular

$$
\int_{-\infty}^{\infty} d \tau \int_{0}^{1} d t\left|\frac{\partial u_{0}}{\partial \tau}\right|_{J_{\infty}}^{2}<\infty
$$

and that satisfies the equation

$$
\left\{\begin{array}{l}
\frac{\partial u}{\partial \tau}+J_{\infty}(\tau, t, u)\left(\frac{\partial u}{\partial t}-X_{H}(u)\right)=0 \\
u(\tau, 0), u(\tau, 1) \in L
\end{array}\right.
$$

on $\mathbb{R} \times[0,1]$. It follows from (2.10) and (2.11) that

$$
\int_{-\infty}^{\infty} \int_{0}^{1}\left|\frac{\partial u}{\partial t}-X_{H}(u)\right|_{J_{\infty}}^{2} d t d \tau<\infty
$$

Hence there exists a sequence $\left\{\tau_{n}\right\}$ with $\tau_{n} \rightarrow \infty$ as $n \rightarrow \infty$ such that

$$
\int_{0}^{1}\left|\dot{z}_{n}-X_{H}\left(z_{n}\right)\right|_{J_{0}}^{2} d t \rightarrow 0 \quad \text { as } n \rightarrow \infty
$$

where $z_{n}:=u\left(\tau_{n}, \cdot\right)$. Here we recall that $J_{\infty}(\tau, \cdot) \equiv J_{0}$ for $\tau$ with $|\tau|$ sufficiently large. Since $H$ has compact support and so $\left|X_{H}\right|$ is bounded, this implies $\left\|\dot{z}_{n}\right\|_{L^{2}}$ is bounded, which in turn implies that $\left\|z_{n}\right\|_{H^{1}}$ is bounded because $z_{n}(0) \in L$ where $L$ is compact. By compactness of the Sobolev embedding $H^{1} \hookrightarrow C^{0}$, taking a subsequence if necessary, $z_{n} \rightarrow z$ in $C^{0}$ for some continuous map $z$ : $[0,1] \rightarrow P$. Then by standard bootstrap arguments, we prove that $z_{n} \rightarrow z$ in $C^{\infty}$ and $z$ satisfies the equation

$$
\left\{\begin{array}{l}
\dot{z}=X_{H}(z) \\
z(0), z(1) \in L
\end{array}\right.
$$


This contradicts to the assumption (2.2) because any such Hamiltonian orbit would give rise to to an intersection point in $L \cap \phi_{H}^{1}(L)$.

Note that Lemma 2.1 and 2.2 hold for any $\bar{J}$ and $H$ that satisfy (2.3) and (2.2) respectively. Therefore we can do the standard Fredholm theory and the genericity arguments with such pairs $(\bar{J}, H)$. We will always carry out this standard genericity argument without further mentioning details, whenever necessary.

Let $H: P \times[0,1] \rightarrow \mathbb{R}$ be a given (generic) Hamiltonian. For generic $\bar{J}=$ $\left\{J_{K}\right\}_{K \in[0,+\infty)}$ satisfying $(2.3)$, we form the parametrized moduli space

$$
\overline{\mathcal{M}}^{0}(\bar{J}, H):=\bigcup_{K \in \mathbb{R}_{+}} \mathcal{M}_{K}^{0}(\bar{J}, H)
$$

which becomes a smooth manifold of dimension $n+1$ with boundary by the parametrized version of the index theorem (2.7), and consider the evaluation map

$$
E v_{0}: \overline{\mathcal{M}}^{0}(\bar{J}, H) \times \mathbb{R} \rightarrow L \times \mathbb{R}_{+} \times \mathbb{R} ; \quad(u, K, \tau) \mapsto(u(\tau, 0), K, \tau) .
$$

We choose smooth embedded paths $\Gamma:[0,1] \rightarrow L \times \mathbb{R}_{+} \times \mathbb{R}$ with

$$
\Gamma(s)=(\gamma(s), K(s), \tau(s))
$$

such that

$$
K(0)=0, \quad \text { and } \quad K_{0} \leq K(1) \leq 2 K_{0}
$$

where $K_{0}$ is the same constant as in Lemma 2.2. Choosing generic $\Gamma$ 's, we can make the map (2.13) transverse to the path $\Gamma$ so that $N_{\Gamma}:=E v_{0}^{-1}(\Gamma)$ becomes a one dimensional manifold with its boundary consisting of

$$
\mathcal{M}_{K(0)}^{0}(\bar{J}, H) \times\{\tau(0)\} \coprod \mathcal{M}_{K(1)}^{0}(\bar{J}, H) \times\{\tau(1)\}
$$

Under the assumption

$$
\phi_{H}^{1}(L) \cap L=\emptyset,
$$

it follows from Lemma 2.2 and 2.3 that the above boundary becomes a single point, i.e, $\left(u_{0}, \tau(0)\right)$ where $u_{0} \equiv \gamma(0)$ is the constant map. Therefore $N_{\Gamma}$ cannot be compact. The only source of non-compactness of $N_{\Gamma}$ lies in the set $\cup_{s \in[0,1]} \mathcal{M}_{K(s)}^{0}(\bar{J}, H)$ which corresponds to bubbling off either $J_{\left(K_{0}, \tau_{0}, t_{0}\right)}$-spheres for some $\left(K_{0}, \tau_{0}, t_{0}\right) \in \mathbb{R}_{+} \times \mathbb{R} \times(0,1)$, or $J_{0}$-holomorphic discs with boundary on $L$. Since $K(1)$ is chosen as in (2.14), in particular bounded, the splitting phenomenon of solutions of (2.4) does not occur. 
Gromov's compactness theorem implies that there exists a sequence $\left\{\left(s_{i}, u_{i}\right)\right\}$ with $s_{i} \rightarrow s_{0}$ and $0<s_{0}<1$ such that $u_{i} \in \mathcal{M}_{K\left(s_{i}\right)}^{0}(\bar{J}, H)$ weakly converges to the cusp curve

$$
u_{\infty}=u_{0}+\sum w_{k}+\sum v_{\ell}
$$

where $u_{0}$ is a solution of (2.4) for $K=K\left(s_{0}\right)$, and $w_{k}$ 's and $v_{\ell}$ 's are $J_{0^{-}}$ holomorphic discs $J_{\left(K\left(s_{0}\right), \tau_{\ell}, t_{\ell}\right)}$-holomorphic spheres for some $\left(\tau_{\ell}, t_{\ell}\right)$ respectively. Here we note that $s_{0}$ cannot be either 0 or 1 , because the corresponding moduli spaces are Fredholm regular. This is because for $s=1, \mathcal{M}_{K(1)}^{0}(\bar{J}, H)=\emptyset$ by the choice of $\Gamma$ and for $s=0, \mathcal{M}_{K(0)}^{0}(\bar{J}, H)=\mathcal{M}_{0}^{0}(\bar{J}, H)$ is regular by Lemma 2.1.

\section{Disjunction energy: proof of Chekanov's theorem}

In this section, we use the set-up given in Section 2 to provide a simple proof of Chekanov's theorem stated in the introduction. In fact, the theorem is an easy consequence of (2.8) and standard bubbling analysis, once we are given the set-up we have made in Section 2.

Theorem 3.1 [Chekanov, C2]. Let $(P, \omega)$ be a tame symplectic manifold and $L \subset(P, \omega)$ be a compact Lagrangian embedding. Then we have

$$
E(L: P, \omega) \geq A_{(L: P, \omega)}>0 .
$$

Proof. Let $H: P \times[0,1] \rightarrow \mathbb{R}$ be a Hamiltonian such that

$$
L \cap \phi_{H}^{1}(L)=\emptyset \text {. }
$$

(If there is no such $H$, the inequality obviously holds because in that case we have $E(L: P, \omega)=+\infty$.) From (2.8), we have

$$
E_{J_{K}}(u) \leq\|H\|
$$

for any solution $u$ of (2.4) with $[u]=0$. In Section 2, we have shown that the parametrized moduli space $\overline{\mathcal{M}}^{0}(\bar{J}, H)$ cannot be compact. Therefore we have sequences $s_{i} \rightarrow s_{0} \in(0,1), K_{i} \rightarrow K_{\infty}$, with $0<K_{\infty}<K_{0}$ and $u_{i} \in \mathcal{M}_{K_{i}}^{0}$ weakly converging to

$$
u_{\infty}=u_{0}+\sum_{k} w_{k}+\sum_{\ell} v_{\ell}
$$

where we have $\left\{w_{k}\right\} \neq \emptyset$ or $\left\{v_{\ell}\right\} \neq \emptyset$. Then we have the following energy inequality

$$
\varlimsup_{i \rightarrow \infty} E_{J_{K_{i}}}\left(u_{i}\right) \geq E_{J_{K_{\infty}}}\left(u_{0}\right)+\sum_{k} E_{J_{0}}\left(w_{k}\right)+\sum_{\ell} E_{J_{\left(K\left(s_{0}\right), \tau_{\ell}, t_{\ell}\right)}}\left(v_{\ell}\right) .
$$


Since there must be at least one $w_{k}$ or $v_{\ell}$, we have

$$
\varlimsup_{i \rightarrow \infty} E_{J_{K_{i}}}\left(u_{i}\right) \geq \min \left\{A_{\left(J_{0}, L: P, \omega\right)}, A_{\left(J_{\left.K\left(s_{0}\right), \tau_{\ell}, t_{\ell}\right)}: P, \omega\right)} \text { 's }\right\}
$$

Note that for any given $\epsilon>0$, we may choose $J=\left\{J_{(K, \tau, t)}\right\}$ arbitrarily $C^{\infty}$-close to the given $J_{0}$ in the genericity argument and so that we have

$$
A_{\left(J_{\left(K\left(s_{0}\right), \tau_{\ell}, t_{\ell}\right)}: P, \omega\right)} \geq A_{\left(J_{0}: P, \omega\right)}-\epsilon .
$$

This last statement can be proven by standard compactness arguments (see [Proposition 4.1, O4] for the proof in a similar context). Combining (3.1)-(3.3), for any given $\epsilon>0$ we have proven

$$
\|H\| \geq \min \left\{A_{\left(J_{0}, L: P, \omega\right)}, A_{\left(J_{0}: P, \omega\right)}\right\}-\epsilon .
$$

Hence comes the proof of

$$
\|H\| \geq \min \left\{A_{\left(J_{0}, L: P, \omega\right)}, A_{\left(J_{0}: P, \omega\right)}\right\}
$$

for any $H$ such that $L \cap \phi_{H}^{1}(L)=\emptyset$. By taking the supremum over $J_{0}$, we have $\|H\| \geq A_{(L: P, \omega)}$ and then by taking the infimum over $H$ with $L \cap \phi_{H}^{1}(L)=\emptyset$, we have finished proof of the theorem.

Note that the proof of this theorem also shows that if $L$ allows a Hamiltonian $H$ such that $\phi_{H}^{1}(L) \cap L=\emptyset$, then we must have

$$
\left.\omega\right|_{\pi_{2}(P, L)} \neq 0
$$

and in particular $\pi_{2}(P, L) \neq 0$. This is also the weaker version of Floer's result on the Arnold conjecture $[\mathrm{F}]$, which had been previously proven by Gromov $[\mathrm{G}]$ : In $[\mathrm{F}]$, it was proven that under the assumption $\left.\omega\right|_{\pi_{2}(P, L)} \equiv 0, \#\left(\phi_{H}^{1}(L) \cap L\right)$ is estimated by the total Betti number of $L$. In fact, Chekanov [C2] proved a stronger result than Theorem 3.1 in that he also estimated the number of intersections $L \cap \phi_{H}^{1}(L)$, when $\|H\|<A_{(L: P, \omega)}$ (see [C2] for details). Using similar ideas used in the present paper, one can simplify Chekanov's proof [C2] to prove this result itself whose details we leave to the interested readers.

\section{References}

[C1] Y. Chekanov, Hofer's symplectic energy and Lagrangian intersections, Publications of the Newton Institute (C .B. Thomas, ed.), vol. 8, Cambridge University Press, 1996, pp. 296-306.

[C2] , Lagrangian intersections, symplectic energy and areas of holomorphic curves, preprint (1995).

[F] A. Floer, Morse theory for Lagrangian intersections, J. Diff. Geom. 28 (1988), 513-547. 
[FHS] A. Floer, H. Hofer, and D. Salamon, Transversality in elliptic Morse theory for the symplectic action, Duke Math. J. 80 (1995), 251-292.

[FHV] A. Floer, H. Hofer, and C. Viterbo, The Weinstein conjecture in $P \times \mathbb{C}^{\ell}$, Math. Z. 203 (1990), 469-482.

[FO] K. Fukaya and K. Ono, Arnold conjecture and Gromov-Witten invariants for general symplectic manifolds, preprint.

[G] M. Gromov, Pseudo-holomorphic curves in symplectic manifolds, Invent. Math. 81 (1985), 307-347.

[H1] H. Hofer, Lüsternik-Schnirelman theory for Lagrangian intersections, Ann. Inst. H. Poincaré, 5(5) (1988), 465-499.

[H2] , On the topological properties of symplectic maps, Proc. Royal Soc. Edinburgh 115 (1990), 25-38.

[LM] F. Lalonde and D. McDuff, The geometry of symplectic energy, Ann. of Math. 141 (1995), 349-371.

[O1] Y. -G. Oh, Removal of boundary singularities of pseudo-holomorphic curves with Lagrangian boundary conditions, Comm. Pure Appl. Math. 45 (1992), 121-139.

[O2] - Floer cohomology of Lagrangian intersections and pseudo-holomorphic discs I, Comm. Pure Appl. Math. 46 (1993), 949-993.

[O3] - On the structure of pseudo-holomorphic discs with totally real boundary conditions, Jour. Geom. Anal (to appear).

[O4] Floer cohomology, spectral sequences and the Maslov class of Lagrangian embeddings, Intern. Math. Res. Notices, No. 7 (1996), 305-346.

[O5] Symplectic topology as the geometry of action functional, I, J. Diff. Geom (to appear).

[O6] Symplectic topology as the geometry of action functional, II, submitted.

[P] L. Polterovich, Symplectic displacement energy for Lagrangian submanifolds, Ergodic Theory Dynamical Systems 13 (1993), 357-367.

Dept. of Mathematics, University of Wisconsin-Madison, Madison, Wi 53706

E-mail address: oh@math.wisc.edu 